\title{
Molecules empowering animals to sense and respond to temperature in changing environments Dominique A Glauser ${ }^{1}$ and Miriam B Goodman ${ }^{2,3}$
}

\begin{abstract}
Adapting behavior to thermal cues is essential for animal growth and survival. Indeed, each and every biological and biochemical process is profoundly affected by temperature and its extremes can cause irreversible damage. Hence, animals have developed thermotransduction mechanisms to detect and encode thermal information in the nervous system and acclimation mechanisms to finely tune their response over different timescales. While temperature-gated TRP channels are the best described class of temperature sensors, recent studies highlight many new candidates, including ionotropic and metabotropic receptors. Here, we review recent findings in vertebrate and invertebrate models, which highlight and substantiate the role of new candidate molecular thermometers and reveal intracellular signaling mechanisms implicated in thermal acclimation at the behavioral and cellular levels.
\end{abstract}

\author{
Addresses \\ ${ }^{1}$ Department of Biology, University of Fribourg, 1700 Fribourg, \\ Switzerland \\ Stanford, CA 94305, USA \\ Corresponding authors: Glauser, Dominique A \\ (dominique.glauser@unifr.ch) and Goodman, Miriam B \\ (mbgoodman@stanford.edu) \\ ${ }^{3}$ Authors listed alphabetically.
}

${ }^{2}$ Department of Molecular and Cellular Physiology, Stanford University,

Variations in ambient temperature have dramatic effects on animal behavior, survival, and reproduction. One dramatic example is the response of a waddle of Emperor penguins to variations in ambient temperature: the colder the temperature, the more likely penguins are to huddle and to huddle more tightly [1]. The social behavior of huddling is essential for thermoregulation, energy conservation, and to complete the breeding cycle. Thus, animals have evolved behavioral and metabolic strategies for minimizing their exposure to rapid fluctuations and for adapting to slow seasonal variations. Such thermal acclimation behaviors depend on the animal's ability to link information provided by specialized thermosensory neurons to appropriate behaviors. Laboratory studies in animals that are amenable to genetic dissection such as fruit flies, nematodes, and mice, have revealed many relevant sensory neurons and thermoreceptor proteins. In this review, we consider candidate thermoreceptor proteins and signaling pathways as well as molecular mechanisms for responding to changing thermal environments, highlighting recent key results and emerging opportunities for discovery in this area.

\section{Molecular basis of thermosensing}

To function as a thermoreceptor, a protein or signaling pathway should exhibit several properties. First, the protein(s) should localize to the presumptive site of temperature-sensing within sensory neurons embedded in tissues subjected to thermal fluctuations [ $\left.2^{\circ}\right]$. Second, the proteins(s) should be required for cellular and behavioral responses to thermal cues. Third, the protein(s) should confer extraordinary temperature sensitivity on heterologous cells and, fourth, protein function should itself be exquisitely temperature-sensitive. Distinguishing ordinary from extraordinary thermal sensitivity is not easy, however. One useful rule of thumb is to focus on proteins or signaling pathways with $Q_{10}$ values that exceed those typical of biochemical reactions such as active transport of ions (ca. 3) across biological membranes [3].

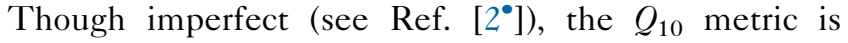
frequently used to assess the effect of temperature on cell function; for ion channels, $Q_{10}$, is commonly estimated from the ratio of the current measured at temperature, $T+10^{\circ} \mathrm{C}$, to that measured at temperature $T$.

Several classes of membrane proteins satisfy at least some of these requirements and are thought to enable thermosensory neurons to detect minute thermal fluctuations. Beyond their common status as membrane proteins, however, these proteins share few, if any sequence or structural motifs, having variable transmembrane domain numbers, and acting either as ion channels or receptors. Whereas thermosensitive ion channels are likely to function as direct thermo-electrical transducers, thermosensitive receptors seem to function indirectly, enabling the temperature-dependent synthesis of second messengers that modulate ordinary ion channels. Receptors linked to temperature sensing have homologs in most organisms. The ion channel proteins have a narrower distribution. While all organisms face thermal fluctuations and membrane proteins proposed as thermoreceptors are widely 
Figure 1

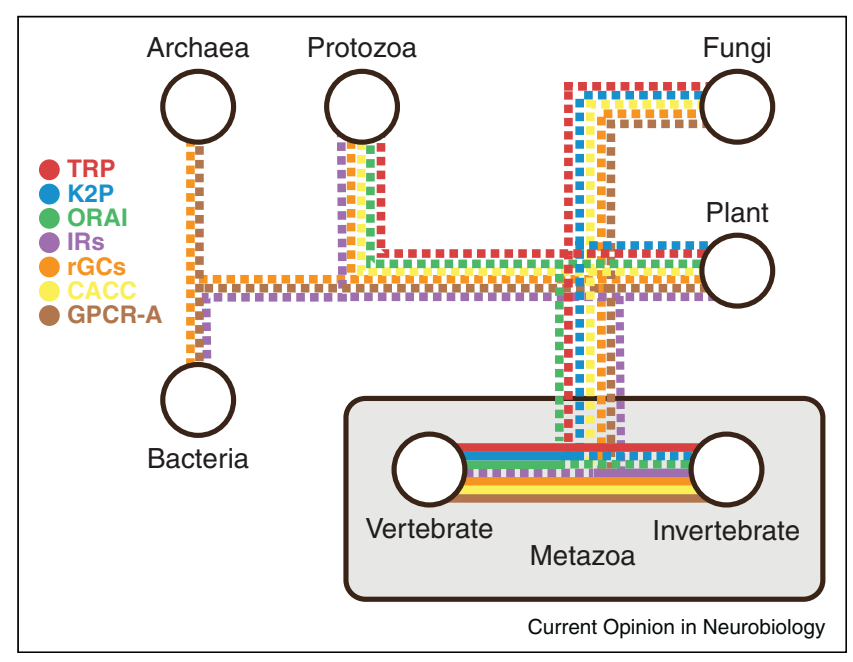

Taxonomy of established and putative molecular thermoreceptors. $\mathrm{TRP}$, transient receptor potential channel proteins; K2P, two-pore $\mathrm{K}$ channels; ORAI, calcium-release activated channels; CACC, calciumactivated chloride channels; rGCs, receptor guanylate cyclases; GCPR, G-protein coupled receptors. Solid lines indicate that experimental support exists for thermoreceptor function of one or more proteins; dashed lines indicate where proteins are present or predicted from genome sequence, but not linked to thermosensation in experimental studies. Information synthesized from investigation of the PFAM database and the following reports:

$\left[15^{\bullet \bullet}, 22^{\bullet \bullet}, 23^{\bullet}, 26,30,32,59,60\right]$. The following PFAM accession numbers were used: TRP, PF06011; K2P, n.a.; ORAI, PF07856; CACC, PF04547; rGC, PF01094; GCPR, CL0192; IR:PF00060.

distributed across phyla $\left[4^{\bullet}\right]$, little is currently known about their function outside of metazoa (Figure 1).

\section{Transient receptor potential (TRP) channels}

Transient receptor potential (TRP) channels are the most extensively studied temperature-gated channels so far. At least eleven mammalian TRP channels display extraordinary and steep temperature dependence $\left[2^{\circ}, 5\right]$, but only a few fulfill the criteria that define a bona fide thermoreceptor protein. TRPV1 and TRPM 8 are two TRP channels widely believed to serve this function. They are expressed in thermosensory neurons, are required for normal behavioral responses to thermal stimulation, and confer extraordinary temperature sensitivity on heterologous cells [6].

Deciphering the role of a given TRP channel in thermosensation is complicated by several factors, including the fact that TRP channels integrate many signals [7] and interact with other proteins, including other TRP channels [8,9]. These factors affect interpretation of experiments performed in vivo and in heterologous systems, which are often somewhat responsive to temperature themselves [10]. Furthermore, certain TRP channels have species-specific functions, exist as multiple isoforms, or control different aspects of thermosensation.
TRPA1 channels illustrate this situation especially well. They are heat sensors in frogs, snakes, chickens and insects, cold sensors in mammals and in the nematode C. elegans and sensors responsive to both cold and heat or temperature-insensitive in zebrafish [10-12]. In mammals, TRPA1 is often co-expressed with TRPV1 and they can form heteromers [8]. In Drosophila, two TRPA channels Painless and dTRPA1 cooperate to generate noxious heat-evoked thermocurrents in class IV polymodal nociceptors [13]. A two-step process was proposed in which heat activates dTRPA1, thereby triggering the initial entry of $\mathrm{Ca}^{2+}$, which in turn activates the $\mathrm{Ca}^{2+}$ dependent Painless channel. Thus, distinct TRPA channels fulfill different roles in thermotransduction.

\section{Other ion channels}

Other thermosensitive channels include the two-pore domain $\mathrm{K}^{+}$channels (K2P), the $\mathrm{Ca}^{2+}$-activated $\mathrm{Cl}^{-}$channel, Anoctamin-1, as well as the STIM1/ORAI1 complex. Their putative role in temperature sensing is covered in several recent reviews $[5,6,14]$. Additionally, a new link between the insect ionotropic receptors (IRs), and temperature sensing was recently uncovered in the dorsal organ cool cells (DOCG) of Drosophila larvae [15 ${ }^{\bullet \bullet}$ ]. The IRs are membrane receptors better studied for their contribution to insect chemosensation and members of the superfamily of membrane proteins that also includes NMDA and AMPA receptors [16]. The DOCG cells are critical for avoiding temperatures below $20^{\circ} \mathrm{C}$, are activated by tiny, transient decreases in temperature [17] and rely on IR21a and IR25a to sense cooling [15 ${ }^{\bullet \bullet}$ ]. Importantly, IR21a confers cool-sensing abilities to other neurons in fruit fly larva. Whereas other members of the Drosophila IR family appear to form odor-gated cation channels in Xenopus oocytes [18], efforts to reconstitute a cooling-activated IR channel complex have yet to succeed. The recent work of Ni, Klein and colleagues [15 $\left.{ }^{\bullet \bullet}\right]$ suggests not only that IR21a and IR25a might function as thermoreceptors, but that other ionotropic receptors might also serve this function.

\section{Single-pass membrane receptors}

Receptor guanylate cyclases (rGCs) are multifunctional proteins consisting of an intracellular enzyme domain that catalyzes the synthesis of cyclic GMP from GTP and a large extracellular domain linked by a single transmembrane domain. Certain rGCs are constitutively active, while others are activated by extracellular peptide ligands. The rGCs present in vertebrate photoreceptors are an example of the former and receptors for atrial natriuretic peptide (ANP) in mammals are examples of the latter [19].

In $C$. elegans, three rGCs, GCY-8, GCY-18 and GCY-23, are required for certain behavioral responses to thermal cues and co-expressed only in the AFD thermosensory neurons that detect minute thermal fluctuations of less 
than $0.05^{\circ} \mathrm{C}$. These three rGCs localize to specialized sensory endings, which appear to contain all of the cellular machinery required to detect thermal cues [20]. Loss of all three proteins eliminates temperature-evoked membrane currents [21], calcium transients [22 $2^{\bullet \bullet}$, and impairs temperature-evoked behaviors. Thus, these three proteins have at least two of the characteristics expected from a thermoreceptor.

Are the AFD-rGC proteins sufficient to confer extraordinary temperature sensitivity on heterologous cells? The work of Takeishi, Yu and colleagues [22 $2^{\bullet \bullet}$ ] answers this question in the affirmative: ectopic expression of all three AFD rGCs together confers extraordinary thermosensitivity on other $G$. elegans sensory neurons. Ectopic expression of GCY-23 alone, but not GCY-8 is sufficient to impart thermosensitivity. GCY-18 expression resulted in calcium signals at high temperatures outside of AFD's normal operating range, providing an opportunity to explore which domain(s) set the thermal threshold. Analysis of chimeric proteins indicates that not only the extracellular and intracellular domains, but also the transmembrane segment influence the thermosensitivity of rGCs-mediated calcium signaling.

The homology between the AFD rGC proteins and other rGCs strongly implies that they catalyze the synthesis of cGMP. Indeed, Singhvi and collaborators [23] have recently shown that $C$. elegans GCY-8 functions as a constitutively active, chloride-inhibited guanylate cyclase in mammalian cells. While it is not yet known how GCY-8 activity depends on temperature, prior analysis of another C. elegans $\mathrm{rGC}$ revealed that warming increases and then decreases GCY-12 activity in mammalian cells [24]. The significance of $\mathrm{Cl}^{-}$inhibition remains obscure, but some intriguing possibilities are emerging. For instance, KCC-3 is a putative $\mathrm{K}-\mathrm{Cl}$ co-transporter expressed in a glial-like cell that surrounds the AFD sensory ending and is required for normal thermotaxis $\left[23^{\circ}, 25^{\circ}\right]$. While loss of KCC-3 dramatically alters calcium signals evoked by a thermal ramp, it does not eliminate such responses [25 $5^{\circ}$. The persistence of warming-evoked calcium signals in $k c c-3$ mutants establishes that this co-transporter is not essential for initiating AFD's response to thermal stimuli in intact, living animals.

Collectively, these studies support the idea that AFD rGCs function as thermoreceptor proteins. Of note, the AFD rGCs are not the only rGCs linked to thermosensation: in mice, the GC-G protein is expressed in thermosensory neurons activated by cooling and functions as a cooling-activated enzyme in heterologous cells [26].

\section{Multi-pass membrane receptors}

Membrane receptors linked to temperature sensing include GR28b(D) and rhodopsin. GR28b(D) is a member of the family of gustatory receptors (GRs) and is encoded by a complex genetic locus gathering several closely related receptors in Drosophila melanogaster fruit flies [27]. GRs are a family of seven-pass membrane proteins linked to chemical sensing in fruit flies and other insects [28]. However, whether they assemble as dimers, function as ion channels, or signal via G-protein mediated pathways remains unclear. GR28b(D) is expressed in three hot cells (HCs), which are peripheral sensory neurons embedded in the antenna. GR28b(D) ectopic expression is sufficient to confer temperature-sensitivity on motor neurons and muscles [29 $9^{\bullet \bullet}$. These findings suggest that GR28b(D) functions as a thermoreceptor protein in Drosophila HCs.

Rhodopsin is a classical $G$ protein-Coupled Receptor (GPCR) known for its central role as a photon detector critical for vision. It belongs to a large family of opsin-like proteins, some of which are expressed outside the nervous system. In fruit fly larvae, disrupting genes encoding rhodopsin [30] and its downstream signaling elements [31] alters thermotaxis behavior. In support of the idea that they have a conserved function in thermotaxis, opsins and downstream signaling elements such as phospholipase $\mathrm{C}$ (PLC) have been linked to sperm thermotaxis in mammals, including humans [32]. Whether or not rhodopsin functions as a bona fide thermoreceptor protein is controversial, however, since it also functions as a highly thermostable photon detector [33]. Additional work is needed to examine this question and to more fully elucidate the role that opsins play in thermotaxis behaviors in animals and in mammalian sperm.

\section{Thermosensory plasticity}

Go for a swim in cool water on a summer day. The water will feel very cold at first, but within minutes, you will acclimate and feel more comfortable. Later, you will be able to detect mild thermal variations while swimming through slightly cooler or warmer streams. Such adaptation is common in sensory systems and influences thresholds for the sensation of noxious heat and cold in animals, including humans [34]. It can involve diverse mechanisms operating in various locations, from primary receptor neurons to the central nervous system, and over various time scales, from seconds to days.

Recent studies in animal models provide insight into the molecular and cellular mechanisms occurring in primary sensory neurons and controlling thermal acclimation in vivo. In a systematic analysis, Brenner and collaborators found that withdrawal responses evoked by both warm and cold stimuli depend on a dynamic temperature 'setpoint', that is modified by past experience in mice [ $\left.35^{\circ \bullet}\right]$. Using genetic knockout and pharmacologic inhibition approaches, they showed that phospholipase C (PLG) activity and TRPM8 are needed for full adaptation to cooler ambient temperatures. Since depletion of membrane PIP2 by the $\mathrm{Ca}^{2+}$-sensitive PLC $\delta$ decreases 


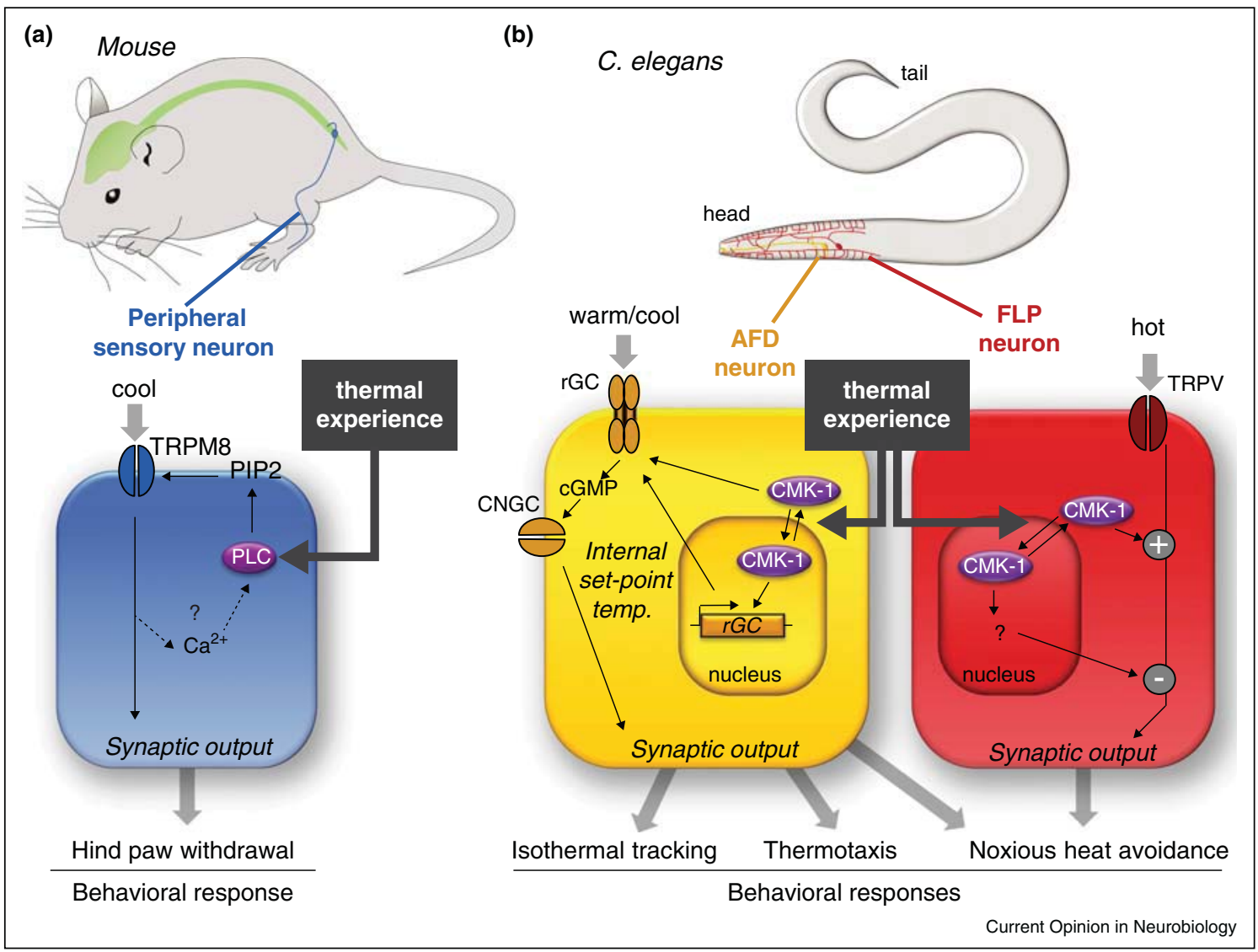

Molecular mechanisms of thermal acclimation in vivo. Schematic representation of recently identified molecular mechanisms underlying thermal acclimation in mice (a) and in the nematode C. elegans (b). TRPM8, transient receptor potential M8; PLC, phospholipase C; PIP2, phosphatidylinositol biphosphate; rGC, receptor guanylate cyclase; cGMP, cyclic guanosine monophosphate; CNGC, cyclic nucleotide gated channel; CMK-1, $\mathrm{Ca}^{2+} /$ calmodulin-dependent protein kinase 1; TRPV, transient receptor potential V.

TRPM8 sensitivity in vitro [36], it is possible that PLC signaling acts directly on TRPM8 activity to mediate adaptation of cold temperature responses. How PLC activation is controlled in this context in vivo is yet unknown, but it is possible that $\mathrm{Ca}^{2+}$ signals could be implicated, thus constituting a relatively direct feedback loop coupling TRPM8 channel activity, which triggers $\mathrm{Ca}^{2+}$ entry, with its own down-regulation (Figure $2 \mathrm{a}$ ).

Additional insights come from studies of temperatureevoked behaviors in $C$. elegans. These animals use noxious heat avoidance to limit damage, thermotaxis to find a preferred temperature in the innocuous temperature range and isothermal tracking to stay close to this preferred temperature [20,37-39]. All three behaviors are modulated by previous temperature experience, implying some sort of memory. Indirect evidence has long suggested that AFD thermosensory neurons could themselves encode the memory of past thermal experience relevant for thermotaxis and isothermal tracking [40,41]. This was demonstrated by Kobayashi and collaborators, showing that cultured AFD neurons harbor all of the necessary machinery to store and update a thermal memory $\left[42^{\circ}\right]$, although ex vivo responses were more variable than in vivo responses. In another study, the same group showed that in worms navigating in a continuous temperature gradient, the combination of motion-imposed thermal variations and short-term adaptation properties produced intermittent activity peaks in AFD [43]. Discrete AFD activity events correlated with turns in well-fed, but not starved animals. Given that starvation suppresses thermotaxis [44], these findings suggest that varying the degree of coupling between the activity of AFD and downstream behavioral responses is a plausible neural mechanism through which feeding state regulates temperature-dependent behavior.

The AFD thermosensory neuron operates in a temperature range that depends on past thermal experience and is modulated by cGMP levels [45]. Thermal re-setting or adaptation takes place in two phases in AFD: a short one (minute timescale) and a long one (hour timescale) [46 $\left.{ }^{\circ}\right]$. 
Both resetting phases require intact CMK-1 signaling and adaptation to warmer temperatures is linked to CMK-1 translocation to the nucleus and regulation of $\mathrm{rGC}$ gene expression (Figure 2b). CaM kinase signaling is well known to couple cell excitation with transcriptional activity [47]. Thus, AFD might use this feedback pathway to ensure long term adaptation (hour timescale) by continuously adjusting thermosensory protein expression levels as a function of its thermo-dependent activity. One could speculate that the expression level of the different relevant rGCs (GCY-8, GCY-18 and GCY-23) or their ratio might affect the thermal set point. In an independent study based on a novel mutagenesis screen, Schild and collaborators also identified the CMK-1 pathway as controlling behavioral plasticity in noxious heat avoidance. This behavior relies on at least two classes of sensory neurons: AFD and the thermo-nociceptor neuron FLP [48]. Of note, CMK-1 has an active role in FLP neurons to adjust the threshold of thermal avoidance [49 ${ }^{\bullet}$ ]. Upon heat pre-exposure, CMK-1 translocates to the nucleus of FLP neurons, where it has an active role to decrease avoidance. Interestingly, cytoplasmic CMK-1 activity has the opposite effect, suggesting a bi-directional switch based on CMK-1 subcellular localization and able to adjust avoidance behaviors (Figure 2b). The pertinent molecular targets downstream of CMK-1 signaling in FLP neurons are not currently known. However, it seems that FLP in $c m k-1$ mutants has at least superficially intact thermo-detection abilities and that CMK-1 signaling might rather adjust the nature or the degree of neurotransmission to secondary neurons. This situation might be reminiscent of the apparent uncoupling between thermosensation and behavior observed upon starvation in AFD [43].

\section{Conclusions}

Signals generated by thermosensory systems are responsible not only for acute responses to thermal fluctuations, but also for the sustained changes in signaling underpinning thermal acclimation. One largely unsolved question is how biological molecules convert temperature, which is a continuous physical property of matter, into useful neural signals.

Among animal thermoreceptor proteins, the thermoTRP channels are probably the most intensively studied. Two models of temperature-dependent gating are frequently discussed-one posits that temperature-dependent channel activation is conferred by specific protein domains, while another states that the activation process reflects temperature-dependent protein unfolding that leads to the exposure of previously buried amino acid residues [7,50]. The currently booming cryo-electron microscopy structural biology approach [51] has provided the first high-resolution structures of two thermo'TRPs [52,53] and such structures are already providing a critical substrate for computational and experimental work.
Still, animal thermoreceptor proteins share few, if any functional domains. Yet, all are membrane proteins - an observation that suggests that the membrane-protein interface could prove critical for their function. In support of this idea, the bacterial membrane protein DesKC responds to temperature-driven variations in membrane thickness and fluidity, biasing the catalytic activity of its cytoplasmic domain toward kinase activity upon cooling and toward phosphatase activity upon heating [54 ${ }^{\bullet \bullet}$. Further analysis of this protein has revealed that one of its transmembrane segments undergoes a conformational switch in response to changes in bilayer thickness [55]. Perhaps an analogous biophysical mechanism is behind the extraordinary temperature-dependence of animal sensory neurons and the diversity of membrane proteins linked to this function in both invertebrates and vertebrates.

Other outstanding questions include whether and how thermoreceptor protein activity is linked to short- and long-term adaptation. During thermal acclimation, for instance, CMK-1 CaM-kinase accumulates in the nuclei of the AFD and FLP thermosensory neurons in $C$. elegans and is presumed to affect gene transcription upon arrival (Figure 2b). But, thermal stimuli alone are insufficient to stimulate the movement of CMK-1, since thermal conditioning does not affect CMK-1's cytoplasmic-nuclear distribution in other neurons $\left[49^{\circ}\right]$. One possibility, consistent with studies of related CaM kinases in mammals [56], is that CMK-1 is sequestered in the cytoplasm under steady-state conditions and released in response to heating-evoked calcium transients, enabling its subsequent nuclear transport. More investigations are needed to understand how thermal information is integrated over time to produce adaptive sensory acclimation via CaM kinase and other pathways.

The complex landscape of molecular signaling pathways that allows animals to respond to temperature in changing environments is starting to take shape. Expanding the work leveraging classical genetic models, as well as exploring a wider spectrum of animals $[57,58]$ and singlecell organisms are all promising avenues to reveal new functional insights into thermosensation. Deepening our understanding of the molecular and cellular biophysics of extraordinary temperature-sensitivity holds the promise of deciphering how animals detect tiny thermal fluctuations, literally extracting signals from thermal noise.

\section{Conflict of interest}

Nothing declared.

\section{Acknowledgements}

Research in the authors' laboratories is supported by grants from the NIH (NS047715 to MBG) and SNSF (BSSGI0_155764 and PP00P3_150681 to DAG). We thank S. Katta for the graphical design used in Figure 1 and current and previous members of our laboratories for discussion about the joys and challenges of studying thermosensation and acclimation. 


\section{References and recommended reading}

Papers of particular interest, published within the period of review, have been highlighted as:

- of special interest

$\bullet$ of outstanding interest

1. Gilbert C, Robertson G, Le Maho Y, Ancel A: How do weather conditions affect the huddling behaviour of emperor penguins? Polar Biol 2008, 31:163-169.

2. Voets T: Quantifying and modeling the temperature-dependent gating of TRP channels. Rev Physiol Biochem Pharmacol 2012 162:91-119.

Review of so-called thermoTRPs and in-depth discussion of $Q_{10}$, thermal thresholds, and activation energy as metrics of extraordinary versus ordinary temperature-dependence.

3. Weiss TF: Cellular Biophysics: Transport. MIT Press; 1996.

4. Sengupta P, Garrity P: Sensing temperature. Curr Biol 2013, 23:R304-R307.

Primer on molecular mechanisms of temperature sensing, including definitions of $Q_{10}$.

5. Vriens J, Nilius B, Voets T: Peripheral thermosensation in mammals. Nat Rev Neurosci 2014, 15:573-589.

6. Palkar R, Lippoldt EK, McKemy DD: The molecular and cellular basis of thermosensation in mammals. Curr Opin Neurobiol 2015, 34:14-19.

7. Hilton JK, Rath P, Helsell CV, Beckstein O, Van Horn WD: Understanding thermosensitive transient receptor potential channels as versatile polymodal cellular sensors. Biochemistry 2015, 54:2401-2413.

8. Akopian AN: Regulation of nociceptive transmission at the periphery via TRPA1-TRPV1 interactions. Curr Pharm Biotechnol 2011, 12:89-94.

9. Weng H-J, Patel Kush N, Jeske Nathaniel A, Bierbower Sonya M, Zou W, Tiwari V, Zheng Q, Tang Z, Mo Gary $\mathrm{CH}$, Wang $\mathrm{Y}$ et al.: Tmem100 is a regulator of TRPA1-TRPV1 complex and contributes to persistent pain. Neuron 2016, 85:833-846.

10. Oda M, Kurogi M, Kubo Y, Saitoh O: Sensitivities of two zebrafish TRPA1 paralogs to chemical and thermal stimuli analyzed in heterologous expression systems. Chem Senses 2016, 41:261-272.

11. Barbagallo B, Garrity PA: Temperature sensation in Drosophila. Curr Opin Neurobiol 2015, 34:8-13.

12. Laursen WJ, Anderson EO, Hoffstaetter LJ, Bagriantsev SN, Gracheva EO: Species-specific temperature sensitivity of TRPA1. Temperature 2015, 2:214-226.

13. Terada S, Matsubara D, Onodera K, Matsuzaki M, Uemura T, Usui T: Neuronal processing of noxious thermal stimuli mediated by dendritic $\mathrm{Ca}(2+)$ influx in Drosophila somatosensory neurons. Elife 2016:5.

14. Schneider ER, Anderson EO, Gracheva EO, Bagriantsev SN: Temperature sensitivity of two-pore (K2P) potassium channels. Curr Top Membr 2014, 74:113-133.

15. Ni L, Klein M, Svec KV, Budelli G, Chang EC, Ferrer AJ,

- Benton R, Samuel AD, Garrity PA: The Ionotropic Receptors IR21a and IR25a mediate cool sensing in Drosophila. Elife 2016:5.

First evidence that the Drosophila ionotropic receptors IR21a and IR25a contribute to the detection of cool temperatures; introduces this family of membrane proteins as potential thermoreceptors.

16. Benton R, Vannice KS, Gomez-Diaz C, Vosshall LB: Variant ionotropic glutamate receptors as chemosensory receptors in Drosophila. Cell 2009, 136:149-162.

17. Klein M, Afonso B, Vonner AJ, Hernandez-Nunez L, Berck M, Tabone CJ, Kane EA, Pieribone VA, Nitabach MN, Cardona A et al.: Sensory determinants of behavioral dynamics in Drosophila thermotaxis. Proc Natl Acad Sci U S A 2015, 112:E220-E229.
18. Abuin L, Bargeton $\mathrm{B}$, Ulbrich $\mathrm{MH}$, Isacoff EY, Kellenberger S, Benton R: Functional architecture of olfactory ionotropic glutamate receptors. Neuron 2011, 69:44-60

19. Kuhn M: Molecular physiology of membrane guanylyl cyclase receptors. Physiol Rev 2016, 96:751-804.

20. Garrity PA, Goodman MB, Samuel AD, Sengupta P: Running hot and cold: behavioral strategies, neural circuits, and the molecular machinery for thermotaxis in C. elegans and Drosophila. Genes Dev 2010, 24:2365-2382.

21. Ramot D, Maclnnis BL, Goodman MB: Bidirectional temperature-sensing by a single thermosensory neuron in $C$. elegans. Nat Neurosci 2008, 11:908-915.

22. Takeishi A, Yu YV, Hapiak VM, Bell HW, O'Leary T, Sengupta P.

- Receptor-type guanylyl cyclases confer thermosensory responses in C. elegans. Neuron 2016, 90:235-244.

This study builds on studies of the contribution of $C$. elegans rGCs to thermosensation and shows that their expression in non-thermosensitive cells is sufficient to confer thermosensitivity. Furthermore, reveals the contribution of multiple rGC protein domains.

23. Singhvi A, Liu B, Friedman CJ, Fong J, Lu Y, Huang XY, Shaham S:

- A glial $\mathrm{K} / \mathrm{Cl}$ transporter controls neuronal receptive ending shape by chloride inhibition of an rGC. Cell 2016.

Together with Ref. [25"], reveals the role of the $C$. elegans glial $\mathrm{K}^{+} / \mathrm{Cl}^{-}$ transporter KCC-3 in controlling AFD thermosensory neuron shape and activity. This study also shows that the GCY-8 rGC implicated in AFD thermosensation is inhibited by $\mathrm{Cl}^{-}$ions.

24. Yu S, Avery L, Baude E, Garbers DL: Guanylyl cyclase expression in specific sensory neurons: a new family of chemosensory receptors. Proc Natl Acad Sci U S A 1997, 94:3384-3387.

25. Yoshida A, Nakano S, Suzuki T, Ihara K, Higashiyama T, Mori I: A

- $\quad$ glial $\mathrm{K}(+) / \mathrm{Cl}(-)$ cotransporter modifies temperature-evoked dynamics in Caenorhabditis elegans sensory neurons. Genes Brain Behav 2016, 15:429-440.

Together with Ref. $\left[23^{\circ}\right.$ ], reveals the role of the $C$. elegans glial $\mathrm{K}^{+} / \mathrm{Cl}^{-}$ transporter KCC-3 in thermosensation and AFD thermosensory neuron shape and activity.

26. Chao YC, Chen CC, Lin YC, Breer H, Fleischer J, Yang RB: Receptor guanylyl cyclase-G is a novel thermosensory protein activated by cool temperatures. EMBO J 2015, 34:294-306.

27. Thorne N, Amrein H: Atypical expression of Drosophila gustatory receptor genes in sensory and central neurons. J Comp Neurol 2008, 506:548-568.

28. Joseph RM, Carlson JR: Drosophila chemoreceptors: a molecular interface between the chemical world and the brain. Trends Genet 2015, 31:683-695.

29. Ni L, Bronk P, Chang EC, Lowell AM, Flam JO, Panzano VC,

•• Theobald DL, Griffith LC, Garrity PA: A gustatory receptor paralogue controls rapid warmth avoidance in Drosophila Nature 2013, 500:580-584.

First evidence that that a Drosophila gustatory receptor could work to detect temperature changes; introduces this family of membrane proteins as potential thermoreceptors.

30. Shen WL, Kwon Y, Adegbola AA, Luo J, Chess A, Montell C: Function of rhodopsin in temperature discrimination in Drosophila. Science 2011, 331:1333-1336.

31. Kwon Y, Shim HS, Wang X, Montell C: Control of thermotactic behavior via coupling of a TRP channel to a phospholipase C signaling cascade. Nat Neurosci 2008, 11:871-873.

32. Perez-Cerezales S, Boryshpolets S, Afanzar O, Brandis A, Nevo R Kiss $\mathrm{V}$, Eisenbach $\mathrm{M}$ : Involvement of opsins in mammalian sperm thermotaxis. Sci Rep 2015, 5:16146.

33. Minke B, Peters M: Cell biology. Rhodopsin as thermosensor? Science 2011, 331:1272-1273.

34. Green BG, Akirav C: Threshold and rate sensitivity of lowthreshold thermal nociception. Eur J Neurosci 2010, 31: 1637-1645.

35. Brenner DS, Golden JP, Vogt SK, Dhaka A, Story GM, Gereau

•• Iv RW: A dynamic set point for thermal adaptation requires 
phospholipase C-mediated regulation of TRPM8 in vivo. Pain 2014, 155:2124-2133.

This study is one of a small number that address the molecular mechanisms of thermosensory adaptation in mice in vivo. It capitalizes on a robust cold acclimation experimental paradigm.

36. Rohacs T, Lopes CM, Michailidis I, Logothetis DE: PI(4,5)P2 regulates the activation and desensitization of TRPM8 channels through the TRP domain. Nat Neurosci 2005, 8: 626-634.

37. Glauser DA: How and why Caenorhabditis elegans uses distinct escape and avoidance regimes to minimize exposure to noxious heat. Worm 2013, 2:e27285.

38. Schild LC, Glauser DA: Dynamic switching between escape and avoidance regimes reduces Caenorhabditis elegans exposure to noxious heat. Nat Commun 2013, 4:2198.

39. Wittenburg N, Baumeister R: Thermal avoidance in Caenorhabditis elegans: an approach to the study of nociception. Proc Natl Acad Sci U S A 1999, 96:10477-10482.

40. Clark DA, Biron D, Sengupta P, Samuel AD: The AFD sensory neurons encode multiple functions underlying thermotactic behavior in Caenorhabditis elegans. J Neurosci 2006, 26:74447451

41. Kimura KD, Miyawaki A, Matsumoto K, Mori I: The C. elegans thermosensory neuron AFD responds to warming. Curr Biol 2004, 14:1291-1295.

42. Kobayashi K, Nakano S, Amano M, Tsuboi D, Nishioka T, Ikeda S,

- Yokoyama G, Kaibuchi K, Mori I: Single-cell memory regulates a neural circuit for sensory behavior. Cell Rep 2016, 14:11-21.

Reveals that $C$. elegans thermosensory neurons in isolation contains the full machinery required to memorize, retrieve, and reset thermal set-points.

43. Tsukada Y, Yamao M, Naoki H, Shimowada T, Ohnishi N, Kuhara A, Ishii S, Mori I: Reconstruction of spatial thermal gradient encoded in thermosensory neuron AFD in Caenorhabditis elegans. J Neurosci 2016, 36:2571-2581.

44. Ramot D, Maclnnis BL, Lee HC, Goodman MB: Thermotaxis is a robust mechanism for thermoregulation in Caenorhabditis elegans nematodes. J Neurosci 2008, 28:12546-12557.

45. Wasserman SM, Beverly M, Bell HW, Sengupta P: Regulation of response properties and operating range of the AFD thermosensory neurons by cGMP signaling. Curr Biol 2011, 21:353-362.

46. Yu YV, Bell HW, Glauser DA, Van Hooser SD, Goodman MB,

- Sengupta P: CaMKI-dependent regulation of sensory gene expression mediates experience-dependent plasticity in the operating range of a thermosensory neuron. Neuron 2014, 84:919-926.

Together with Ref. [49], reveals the central role of CaM kinase-I signaling and nucleo-cytoplasmic shuttling in controlling thermal sensory adaptation in C. elegans, with a focus on the response to innocuous temperatures.

47. Wayman GA, Lee YS, Tokumitsu H, Silva AJ, Soderling TR: Calmodulin-kinases: modulators of neuronal development and plasticity. Neuron 2008, 59:914-931.
48. Liu S, Schulze E, Baumeister R: Temperature- and touchsensitive neurons couple CNG and TRPV channel activities to control heat avoidance in Caenorhabditis elegans. PLoS One 2012, 7:e32360.

49. Schild LC, Zbinden L, Bell HW, Yu YV, Sengupta P, Goodman MB,

- Glauser DA: The balance between cytoplasmic and nuclear CaM kinase-1 signaling controls the operating range of noxious heat avoidance. Neuron 2014, 84:983-996.

Together with Ref. [46 ${ }^{\circ}$, reveals the central role of CaM kinase-I signaling and nucleo-cytoplasmic shuttling in controlling thermal sensory adaptation in $C$. elegans, with a focus on the response to noxious temperatures.

50. Qin F: Demystifying thermal channels: driving a channel both forwards and backwards with a single gear? Biophys J 2013, 104:2118-2120.

51. Bai XC, McMullan G, Scheres SH: How cryo-EM is revolutionizing structural biology. Trends Biochem Sci 2015 , 40:49-57.

52. Paulsen CE, Armache JP, Gao Y, Cheng Y, Julius D: Structure of the TRPA1 ion channel suggests regulatory mechanisms. Nature 2015, 520:511-517.

53. Cao E, Liao M, Cheng Y, Julius D: TRPV1 structures in distinct conformations reveal activation mechanisms. Nature 2013 , 504:113-118.

54. Cybulski LE, Martín M, Mansilla MC, Fernández A, de Mendoza D:

- Membrane thickness cue for cold sensing in a bacterium. Curr Biol 2010, 20:1539-1544.

Shows that DesK protein responds to temperature-induced changes in membrane thickness, revealing a biophysical mechanism that might be shared by the diverse membrane proteins linked to temperature sensation.

55. Cybulski LE, Ballering J, Moussatova A, Inda ME, Vazquez DB, Wassenaar TA, de Mendoza D, Tieleman DP, Killian JA: Activation of the bacterial thermosensor DesK involves a serine zipper dimerization motif that is modulated by bilayer thickness. Proc Natl Acad Sci U S A 2015, 112:6353-6358.

56. Cohen SM, Li B, Tsien RW, Ma H: Evolutionary and functional perspectives on signaling from neuronal surface to nucleus. Biochem Biophys Res Commun 2015, 460:88-99.

57. Peng G, Shi X, Kadowaki T: Evolution of TRP channels inferred by their classification in diverse animal species. Mol Phylogenet Evol 2015, 84:145-157.

58. Gracheva EO, Bagriantsev SN: Evolutionary adaptation to thermosensation. Curr Opin Neurobiol 2015, 34:67-73.

59. González W, Valdebenito B, Caballero J, Riadi G, Riedelsberger J, Martínez G, Ramírez D, Zúñiga L, Sepúlveda FV, Dreyer I et al:: K2P channels in plants and animals. Pflügers Archiv - Eur $J$ Physiol 2015, 467:1091-1104.

60. Collins SR, Meyer T: Evolutionary origins of STIM1 and STIM2 within ancient Ca2+ signaling systems. Trends Cell Biol 2011, 21:202-211. 